\title{
Kopfschmerzen breiten sich unter jungen Erwachsenen aus
}

Immer mehr junge Erwachsene leiden unter Kopfschmerzen. Fast jeder zweite Jugendliche greift systematisch nach Schmerzmitteln.

Der Anteil der 18- bis 27-Jährigen mit Kopfschmerzdiagnosen ist zwischen 2005 und 2015 um 42\% gestiegen. Das geht aus dem aktuellen Barmer Arztreport hervor. Demnach leiden inzwischen 1,3 Millionen junge Erwachsene an Kopfschmerzen, 400.000 mehr als noch im Jahr 2005. Die Ursache dieses Anstieg ist jedoch unklar.

Einer repräsentativen Umfrage der Barmer GEK zufolge nehmen bereits $40 \%$ der Kinder und Jugendlichen zwischen neun und 19 Jahren nach eigenen Angaben Medikamente ein, wenn sie Kopfschmerzen haben. Davon bekämpften $42 \%$ den Schmerz sogar jedes Mal mit Arzneimitteln.
Der starke Anstieg der Kopfschmerzdiagnosen bei jungen Erwachsenen sei umso bedenklicher vor dem Hintergrund, dass die Zahl der Diagnosen über alle Altersklassen „nur“ um 12,4\% zugenommen habe. „Ganz sicher haben noch viel mehr junge Menschen mit Kopfschmerz zu kämpfen, als uns aus ärztlichen Diagnosen bekannt ist. Doch diese Gruppe geht tendenziell seltener zum Arzt, weswegen wir sie auf anderem Wege erreichen müssen“, kommentierte Prof. Christoph Straub, Vorstandschef der Barmer.

Dem Arztreport der Krankenkasse zufolge ist auch die Verordnungsrate von Migränemitteln beunruhigend. Denn diese sei bei den 18- bis 27-Jährigen in der

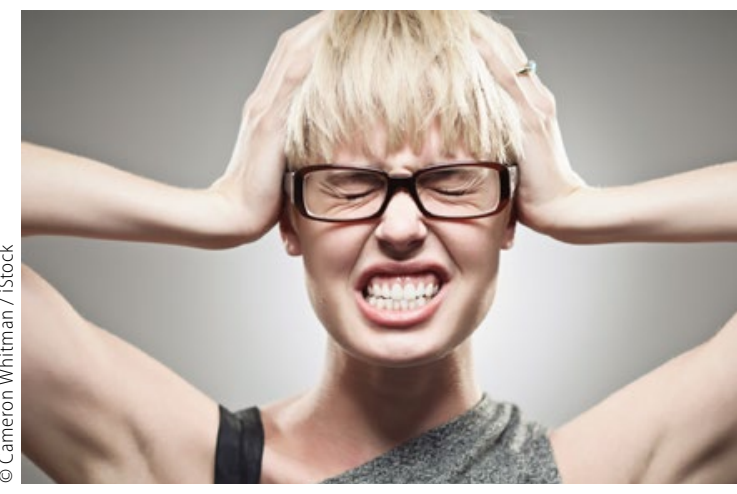

Junge Erwachsene leiden häufiger an

Kopfschmerzen als noch im Jahr 2005.

Zeit von 2005 bis 2015 um ganze 58\% gestiegen. Über alle Altersklassen hinweg betrachtet gab es lediglich einen Anstieg um $9,9 \%$.

\section{Orale Kortikosteroide lindern die Symptome bei Halsschmerzen}

Patienten mit Halsschmerzen profitieren von der einmaligen Behandlung mit einem Kortikosteroid zusätzlich zur Standardtherapie. Das bestätigen die Ergebnisse einer aktuellen Metaanalyse.

Anlass für die Metaanalyse waren die Ergebnisse der TOAST-Studie (Teatment Options without Antibiotics for Sore Throat), an der mehr als 550 Patienten mit Halsschmerzen teilgenommen hatten. Ihre Hausärzte hatten ihnen kein Antibiotikum, sondern Dexamethason verordnet, worauf die Patienten von einer deutlichen Symptomlinderung berichtet hatten. Dr. Behnam Sadeghirad, McMaster-Universtität in Hamilton, Kanada, und seine Kollegen haben nun Daten von insgesamt zehn randomisierten kontrollierten Studien mit mehr als 1400 Patienten ausgewertet. Die Patienten klagten über Halsschmerzen, hatten schmerzhafte Schluckbeschwerden oder eine Pharyngitis. Die Dexamethason-Dosis lag für Erwachsene bei $10 \mathrm{mg}$ und für Kinder bei $0,6 \mathrm{mg} / \mathrm{kg} \mathrm{KG}$ (maximal $10 \mathrm{mg}$ ), einmalig oral.

\section{Mit Steroid früher schmerzfrei}

Wie Sadeghirad und seine Kollegen berichten, war die Wahrscheinlichkeit der Symptomlinderung nach 24 Stunden bei Patienten mit Dexamethasonbehandlung (10 mg) im Vergleich zu Patienten mit einem Scheinpräparat mehr als verdoppelt (relatives Risiko [RR]: 2,2; 95\%-Konfi- denzinterall [KI]: 1,2-4,3). Und die Wahrscheinlichkeit, nach 48 Stunden schmerzfrei zu sein, war um den Faktor 1,5 höher (RR: 1,5; 95\%-KI: 1,3-1,8). Unter Verwendung der visuellen Analogskala (max. zehn Punkte) betrug der Unterschied der absoluten Schmerzlinderung in acht der zehn Studien nach 24 Stunden 1,3 Punkte.

Nach Angaben der Wissenschaftler bestätigen die Ergebnisse ihrer Metaanalyse die Resultate früherer, allerdings kleinerer systematischer Studien. Übereinstimmend kommen alle Studien zu dem Ergebnis, dass Kortikosteroide innerhalb von 48 Stunden die Schmerzen bei Halsschmerzen reduzieren.

ple 